\title{
Virtual Ophthalmology Fellowship Interviews: Perceptions of U.S. Ophthalmology Fellowship Applicants in 2020
}

\author{
Samir N. Patel, MD ${ }^{1}$ \\ Elliot G. Cherkas, BS² \\ Michael A. Klufas, MD ${ }^{1}$ \\ ${ }^{1}$ Retina Service, Wills Eye Hospital, Mid Atlantic Retina, Department \\ of Ophthalmology, Thomas Jefferson University, Philadelphia, \\ Pennsylvania \\ 2 Sidney Kimmel Medical College, Thomas Jefferson University, \\ Philadelphia, Pennsylvania \\ ${ }^{3}$ Glaucoma Service, Wills Eye Hospital, Thomas Jefferson University, \\ Philadelphia, Pennsylvania \\ ${ }^{4}$ Cornea Service, Wills Eye Hospital, Thomas Jefferson University, \\ Philadelphia, Pennsylvania
}

Charlotte N. Shields, BS ${ }^{2}$

Rebecca R. Soares, MD ${ }^{1}$

John W. Hinkle, MD ${ }^{1}$ Reza Razeghinejad, MD ${ }^{3}$ Kristin M. Hammersmith, MD ${ }^{4}$ Brenton D. Finklea, MD ${ }^{4}$ Carol L. Shields, MD ${ }^{5}$ Michael N. Cohen, MD ${ }^{1}$ M. A. Khan, MD ${ }^{1} \quad$ Ajay E. Kuriyan, MD ${ }^{1}$

\author{
Address for correspondence Michael A. Klufas, MD, Retina Service, \\ Wills Eye Hospital, Mid Atlantic Retina, Department of \\ Ophthalmology, Thomas Jefferson University, 840 Walnut Street, \\ Suite 1020, Philadelphia, PA 19107 \\ (e-mail: mklufas@midatlanticretina.com). \\ ${ }^{5}$ Ocular Oncology Service, Wills Eye Hospital, Thomas Jefferson \\ University, Philadelphia, Pennsylvania
}

J Acad Ophthalmol 2021;13:e102-e107.

received

February 3, 2021 accepted after revision April 3, 2021
Objective This study aimed to evaluate the experiences and preferences of ophthalmology fellowship applicants utilizing a virtual interview format.

Design Present study is a cross-sectional study.

Subjects All fellowship applicants to Wills Eye Hospital during 2020 to 2021 application cycle were included.

Methods A nonvalidated, online survey was conducted, and surveys were distributed at the conclusion of the interview process after rank list submission.

Main Outcome Measures Applicant demographics, application submissions, interview experiences, financial considerations, and suggestions for improvement of the virtual interview process were the primary outcomes of this cross-sectional study.

Results Survey responses were received from 68 fellowship applicants (34\% response rate). Thirty (44\%) applicants preferred in-person interviews, 25 (36\%) preferred virtual interviews, and 13 (19\%) would like to prefer the option to choose either. Fifty-five of 68 (80\%) applicants attended the same range of interviews for which they received interview invitations. Reduced costs were reported as the highest ranked strength of virtual interviews in $44(65 \%)$ applicants, with a majority of respondents $(68 \%)$ spending less than U.S. $\$ 250$ throughout the entire process. The highest ranked limitation for virtual interviews was limited exposure to the culture/environment of the program in $20(29 \%)$ respondents. On a scale of 0 to 100 , the mean (standard deviation [SD]) satisfaction level with the fellowship application process was $74.6(18.3)$ and mean (SD) perceived effectiveness levels of virtual interviews was 67.4 (20.4).

virtual interviews

- medical education

DOI https://doi.org/ $10.1055 / \mathrm{s}-0041-1733938$. ISSN 2475-4757.

\footnotetext{
(C) 2021. The Author(s).

This is an open access article published by Thieme under the terms of the Creative Commons Attribution-NonDerivative-NonCommercial-License, permitting copying and reproduction so long as the original work is given appropriate credit. Contents may not be used for commercial purposes, or adapted, remixed, transformed or built upon. (https://creativecommons.org/ licenses/by-nc-nd/4.0/) Thieme Medical Publishers, Inc., 333 Seventh Avenue, 18th Floor, New York, NY 10001, USA
} 
Conclusion Respondents were generally satisfied with virtual interviews and noted reduced costs and increased ability to attend more fellowship interviews as the strengths of the virtual interview format. Limited exposure to the culture/environment of the program was cited as the most important limitation.

The novel coronavirus disease 2019 (COVID-19) pandemic has necessitated rapid advancements in telemedicine and teleeducation over the past year. ${ }^{1,2}$ Given travel restrictions and infectious transmission considerations, many medical schools, residency programs, and fellowship programs have recently transitioned to virtual interviews out of necessity and as a result of these concerns. ${ }^{3-9}$

Multiple recent, survey-based studies have been conducted to understand the impact of virtual interviews on applicants and program directors (PDs) for various nonophthalmology residency and fellowship programs. $3,5,8,10$ One study of cardiothoracic fellowship applicants and PDs found that $79 \%$ of PDs and $55 \%$ of applicants believed that virtual interviews should be offered in the future. However, only $15 \%$ of PDs and $20 \%$ of applicants agreed that virtual interviews should be the only option offered to candidates. ${ }^{5}$ Similarly, another study of general surgery oncology fellows found that $83 \%$ of PDs and $79 \%$ of candidates felt comfortable creating their rank list following a virtual interview.

There is no data, to our knowledge, on the perceptions of ophthalmology fellowship applicants toward the virtual interview process. The purpose of this study was to evaluate the impact of the virtual interview process for ophthalmology fellowship applicants during the COVID-19 pandemic.

\section{Methods}

This prospective cross-sectional study was approved by the Wills Eye Hospital Institutional Review Board. Data were collected in accordance with Health Insurance Portability and Accountability Act guidelines, and the study conformed to the tenets of the Declaration of Helsinki. All study participants provided written informed consent.

A nonvalidated web-based survey was created using SurveyMonkey (SVMK Inc.; San Mateo, CA) for ophthalmology fellowship applicants for the 2020 to 2021 San Francisco (SF) Match fellowship application cycle.

All fellowship applicants ( $n=199)$ to six Wills Eye Hospital (Philadelphia, PA) fellowship programs (Academic Global Ophthalmology, Cornea, Glaucoma, Ocular Oncology, Vitreoretinal Surgery, and Neuroophthalmology) were included. The survey was electronically administered via e-mail on December 11, 2020, a day after the SF Match rank list submission deadline (December 10, 2020). Four reminder e-mails were sent ( December 17, 2020, December 23, 2020, December 28, 2020 , and January 4,2021), and the survey closed on January 9 , 2021. The survey included 22 questions regarding demographic information, the number of programs respondents applied to and interviewed with, monetary costs, virtual interview platform used, satisfaction with the virtual process, perceived strengths and limitations of the virtual interview, and technological difficulties on interview day (-Supplemental Appendix; available in the online version). All data were analyzed using statistical software (IBM SPSS 25 Statistics, Armonk, NY). Statistical significance was considered to be a two-sided $p$-value $<0.05$.

\section{Results}

\section{Applicant Demographics}

Of 199 applicants surveyed, survey responses were obtained from 68 applicants (34\% response rate). Baseline demographics are reported in - Table 1 . Thirty of 68 (44\%) reported their age between 26 and 30, and 33 (49\%) reported age between 31 and 35. Thirty-nine of 68 (57\%) reported male sex, and 9 of 68 (13\%) identified as an underrepresented minority. The majority of applicants were from a residency program/affiliated institution in the Northeast $(n=24$; [35\%]), followed by the Southern United States $(n=17$; [25\%]), Midwestern United States ( $n=12$; [18\%]), Western United States $(n=8 ;[12 \%])$, and Outside the United States lower 48 states $(n=7 ;[10 \%])$. Of the 68 respondents, fellowship application programs included $1(2 \%)$ for Academic Global Ophthalmology, 22 (32\%) for Cornea, 9 (13\%) for Glaucoma, 4 (6\%) for Ocular Oncology, 31 (46\%) for Retina, and 1 (2\%) for other (Oculoplastics).

\section{Application Cycle Process}

Of the 68 respondents, 33 (49\%) applied to more than 30 fellowship programs. Fifty-five of 68 (80\%) respondents attended the same range of interviews for which they received interview invitations, with 34 (50\%) attending more than 15 fellowship interviews, and 13 (19\%) attending more than 20 fellowship interviews (-Table 2 ). Of 49 respondents with less than 20 interviews, 47 (96\%) attended the same range of interviews for which they received interview invitations.

Forty-six of 68 applicants (68\%) reported that the overall cost of their fellowship interviews was less than U.S. \$250, whereas 10 (15\%) reported overall costs greater than U.S. $\$ 1,000$.

\section{Applicant Perceptions on Virtual Interviews}

On a scale of 0 to 100 , the mean (standard deviation [SD]) satisfaction level with the fellowship application process was 74.6 (18.3), and the mean (SD) perceived effectiveness levels of virtual interviews were 67.4 (20.4). When asked regarding future preferences, 30 (44\%) preferred in-person interviews, 25 (36\%) preferred virtual interviews, and 13 (19\%) participants preferred the option to choose either. Applicant perceived strengths (scale: 1-6) and limitations (scale: 1-7) of the virtual interview process are reported 
Table 1 Baseline characteristics of survey respondents

\begin{tabular}{|c|c|c|c|}
\hline $\begin{array}{l}\text { Baseline } \\
\text { demographics }\end{array}$ & & $n$ & $\%$ \\
\hline \multirow[t]{6}{*}{$\begin{array}{l}\text { Fellowship } \\
\text { program }\end{array}$} & $\begin{array}{l}\text { Academic Global } \\
\text { Ophthalmology }\end{array}$ & 1 & 1.5 \\
\hline & Cornea & 22 & 32.4 \\
\hline & Glaucoma & 9 & 13.2 \\
\hline & Ocular Oncology & 4 & 5.9 \\
\hline & Retina & 31 & 45.6 \\
\hline & Other (Oculoplastics) & 1 & 1.5 \\
\hline \multirow[t]{5}{*}{ Age $(y)$} & $<25$ & 0 & 0.0 \\
\hline & $26-30$ & 30 & 44.1 \\
\hline & $31-35$ & 33 & 48.5 \\
\hline & $36-40$ & 3 & 4.4 \\
\hline & $41-45$ & 2 & 2.9 \\
\hline \multirow[t]{2}{*}{ Gender } & Male & 39 & 57.4 \\
\hline & Female & 29 & 42.7 \\
\hline \multirow{5}{*}{$\begin{array}{l}\text { Residency } \\
\text { location (in the } \\
\text { United States) }\end{array}$} & Northeast & 24 & 35.3 \\
\hline & Midwest & 12 & 17.7 \\
\hline & Southern & 17 & 25.0 \\
\hline & Western & 8 & 11.8 \\
\hline & $\begin{array}{l}\text { Outside the United } \\
\text { States lower } 48 \text { states }\end{array}$ & 7 & 10.3 \\
\hline \multicolumn{4}{|c|}{ Fellowship application process } \\
\hline \multirow{7}{*}{$\begin{array}{l}\text { Number of } \\
\text { fellowship } \\
\text { applications }\end{array}$} & $<5$ & 6 & 8.8 \\
\hline & $5-9$ & 6 & 8.8 \\
\hline & $10-14$ & 4 & 5.9 \\
\hline & $15-19$ & 7 & 10.3 \\
\hline & $20-24$ & 6 & 8.8 \\
\hline & $25-29$ & 6 & 8.8 \\
\hline & $>30$ & 33 & 48.5 \\
\hline \multirow{7}{*}{$\begin{array}{l}\text { Number of } \\
\text { fellowship } \\
\text { invitations }\end{array}$} & $<5$ & 7 & 10.3 \\
\hline & $5-9$ & 13 & 19.1 \\
\hline & $10-14$ & 12 & 17.7 \\
\hline & $15-19$ & 17 & 25.0 \\
\hline & $20-24$ & 6 & 8.8 \\
\hline & $25-29$ & 8 & 11.8 \\
\hline & $>30$ & 5 & 7.4 \\
\hline \multirow{7}{*}{$\begin{array}{l}\text { Number of } \\
\text { fellowship } \\
\text { interviews } \\
\text { attended }\end{array}$} & $<5$ & 7 & 10.3 \\
\hline & $5-9$ & 13 & 19.1 \\
\hline & $10-14$ & 14 & 20.6 \\
\hline & $15-19$ & 21 & 30.9 \\
\hline & $20-24$ & 6 & 8.8 \\
\hline & $25-29$ & 4 & 5.9 \\
\hline & $>30$ & 3 & 4.4 \\
\hline
\end{tabular}

Table 1 (Continued)

\begin{tabular}{|c|c|c|c|}
\hline $\begin{array}{l}\text { Baseline } \\
\text { demographics }\end{array}$ & & $n$ & $\%$ \\
\hline \multirow{5}{*}{$\begin{array}{l}\text { Estimated } \\
\text { monetary cost } \\
\text { for all virtual } \\
\text { fellowship } \\
\text { interviews } \\
\text { (U.S. \$) }\end{array}$} & $0-250$ & 46 & 67.7 \\
\hline & $251-500$ & 7 & 10.3 \\
\hline & $501-1,000$ & 5 & 7.4 \\
\hline & $1,001-5,000$ & 9 & 13.2 \\
\hline & $>5,000$ & 1 & 1.5 \\
\hline \multirow{4}{*}{$\begin{array}{l}\text { Location of } \\
\text { virtual } \\
\text { fellowship } \\
\text { interviews }\end{array}$} & Home & 49 & 72.1 \\
\hline & Workplace & 16 & 23.5 \\
\hline & Library & 1 & 1.5 \\
\hline & Other (please specify) & 2 & 2.9 \\
\hline \multicolumn{4}{|c|}{ Applicant preferences } \\
\hline \multirow{3}{*}{$\begin{array}{l}\text { Fellowship } \\
\text { interview } \\
\text { format }\end{array}$} & In-person interview & 30 & 44.1 \\
\hline & Virtual interview & 25 & 36.8 \\
\hline & $\begin{array}{l}\text { Option to choose } \\
\text { either }\end{array}$ & 13 & 19.1 \\
\hline \multirow{4}{*}{$\begin{array}{l}\text { Virtual } \\
\text { interview } \\
\text { platform }\end{array}$} & Zoom & 65 & 95.6 \\
\hline & Microsoft Teams & 1 & 1.5 \\
\hline & Google Hangout & 1 & 1.5 \\
\hline & Other (please specify) & 1 & 1.5 \\
\hline \multirow{5}{*}{$\begin{array}{l}\text { Optimal length } \\
\text { of time for each } \\
\text { video interview } \\
\text { (min) }\end{array}$} & 10 & 4 & 5.9 \\
\hline & 15 & 37 & 54.4 \\
\hline & 20 & 24 & 35.3 \\
\hline & 30 & 2 & 2.9 \\
\hline & 45 & 1 & 1.5 \\
\hline
\end{tabular}

in - Table 3. Reduced costs were reported as the highest ranked strength in 44 of 68 (65\%) respondents with a mean (SD) of 5.5 (1.1). The highest ranked limitation was limited exposure to the culture/environment of the program in 20 (29\%) respondents with a mean (SD) of 5.4 (1.6).

Additionally, respondents reported that speaking to current trainees at the program was the most helpful resource for learning about the program $(n=34 ;[50 \%])$, followed by speaking with mentors $(n=15 ;[22 \%])$.

Participants most commonly indicated that the optimal virtual interview length was 15 minutes $(n=37$ [54\%]), followed by 20 ( $n=24$ [35\%]), $10(n=4$ [6\%]), 30 minutes $(n=2[3 \%])$, and 45 minutes $(n=1[2 \%])$. Regarding virtual interview platforms, respondents used Zoom $(n=67$ [99\%]) most frequently, followed by Microsoft Teams ( $n=17$ [25\%]), SF Match $(n=11[16 \%])$, and Google Hangout $(n=6[9 \%])$. The majority of participants indicated that their preferred virtual platform was Zoom ( $n=65$ [96\%]).

\section{Discussion}

The concept of virtual residency and fellowship interviews has been a topic of interest prior to the COVID-19 pandemic. ${ }^{11}$ In 
Table 2 Association between number of fellowship interview invitations and number of interviews attended by applicants

\begin{tabular}{|c|c|c|c|c|c|c|c|c|c|}
\hline & \multicolumn{9}{|c|}{ Number of Interview Invitations ( $n$ ) } \\
\hline & & $<5$ & $5-9$ & $10-14$ & $15-19$ & $20-24$ & $25-29$ & $>30$ & Total \\
\hline \multirow[t]{8}{*}{ Number of interviews attended $(n)$} & $<5$ & 7 & & & & & & & 7 \\
\hline & $5-9$ & & 13 & & & & & & 13 \\
\hline & $10-14$ & & & 12 & 2 & & & & 14 \\
\hline & $15-19$ & & & & 15 & 5 & & 1 & 21 \\
\hline & $20-24$ & & & & & 1 & 4 & 1 & 6 \\
\hline & $25-29$ & & & & & & 4 & & 4 \\
\hline & $>30$ & & & & & & & 3 & 3 \\
\hline & Total & 7 & 13 & 12 & 17 & 6 & 8 & 5 & 68 \\
\hline
\end{tabular}

support of such a format, Bird et al noted that virtual interviews "provide different information than what is currently available from academic metrics and ... may give program directors an opportunity to widen the skill set of applicants they invite to in-person interviews." ${ }^{12}$ The COVID-19 pandemic accelerated this interest into a necessity, as social distancing guidelines, travel restrictions, safety concerns for applicants, and faculty required a remote or virtual format in many cases. Indeed, several national organizations, including the Association of American Medical Colleges (AAMC), Accreditation Council for Graduate Medical Education (ACGME), and Association for University Professors of Ophthalmology (AUPO), have strongly encouraged or required virtual interview formats for the 2020 application year. ${ }^{13}$

In this study, we sought to assess the experience and preferences of ophthalmology fellowship applicants utilizing the virtual interview format. The greatest strength of the virtual interview process for ophthalmology fellowship applicants was reduced costs. Indeed, in this survey, 46 (68\%) reported that the overall cost of their fellowship interviews was less than U.S. $\$ 250$, whereas prior studies have reported a mean total cost of U.S. $\$ 5,500$ for vitreoretinal fellowship applicants. ${ }^{14}$ Other nonophthalmology studies have noted that using virtual interviews saved both applicants and programs over U.S. \$500 per applicant, ${ }^{15,16}$ specifically by reducing travel and meal costs, while another argued that virtual interviews saved time for both parties. ${ }^{17}$ These savings may have important implications, as a prior study of ophthalmology residency applicants reported that $68 \%$ had to obtain additional funds from family, loans, or credit cards to afford their in-person interviews. ${ }^{18}$ Additionally, as noted by Hariton et al, these high

Table 3 Perceived strengths and limitations of applicants to virtual fellowship interviews

\begin{tabular}{|c|c|c|}
\hline & Mean (SD) & Mode (range) \\
\hline \multicolumn{3}{|l|}{$\begin{array}{l}\text { Strengths of virtual format }{ }^{1} \\
\text { (Scale: } 1=\text { smallest strength to } 6=\text { largest strength) }\end{array}$} \\
\hline Reduced costs & $5.5(1.1)$ & $6(1-6)$ \\
\hline Shorter interview day schedule & $3.6(1.5)$ & $5(1-6)$ \\
\hline Less pressure during interview & $2.1(1.1)$ & $2(1-6)$ \\
\hline Ability to interview at more fellowship programs & $3.4(1.3)$ & $3(1-6)$ \\
\hline Greater scheduling flexibility & $4.2(1.3)$ & $5(1-6)$ \\
\hline \multicolumn{3}{|l|}{$\begin{array}{l}\text { Limitations of virtual format }^{2} \\
\text { (Scale: } 1=\text { smallest limitation to } 7=\text { largest limitation) }\end{array}$} \\
\hline Limited exposure to faculty & $3.4(1.9)$ & $3(1-7)$ \\
\hline Limited exposure to fellows & $3.8(1.8)$ & $3(1-7)$ \\
\hline Limited exposure to the culture/environment of the program & $5.4(1.6)$ & $7(1-7)$ \\
\hline Inability to physically tour the facilities & $4.3(1.7)$ & $6(1-7)$ \\
\hline Limited exposure to the geographic location/city & $4.5(2.0)$ & $6(1-7)$ \\
\hline Limited exposure to details of the program structure (i.e., call schedule and rotation blocks) & $2.3(1.4)$ & $1(1-6)$ \\
\hline Not enough opportunities to exhibit an applicant's strengths to the program & $3.3(1.8)$ & $2(1-6)$ \\
\hline Difficulty making interpersonal connections via virtual interview & $4.4(2.0)$ & $7(1-7)$ \\
\hline
\end{tabular}

Abbreviation: SD, standard deviation. 
personal costs highlight socioeconomic inequalities, favoring those who can afford the extensive travel-related expenses. ${ }^{19}$ By reducing interview-related expenses, programs may help applicants to attend more interview invitations, and help level the playing field for applicants who may be unable to afford attending multiple in-person interviews around the country.

Study respondents also reported greater schedule flexibility as a major benefit of the virtual interview season. One study evaluating ophthalmology fellowship match outcomes from 2012 to 2017 reported that applicants submitted a mean (SD) of 20 (16.6) applications, received 7 (5.4) inperson interview invites, and ranked 7 (5.3) programs. ${ }^{20}$ In this study, 33 (49\%) applicants applied to more than 30 fellowship programs with 34 (50\%) applicants attending more than 15 fellowship interviews. Furthermore, 80\% $(n=55)$ of respondents attended the same range of interviews for which they received interview invitations. Collectively, these findings suggest that applicants are attending more fellowship interviews with the virtual format compared with previous application cycles. Prior studies have reported that one of the most significant barriers to attending an in-person interview has been navigating issues with dates and locations. ${ }^{18}$ With virtual interviews, an applicant could perform multiple interviews from one location, minimizing the need for travel. Additionally, this virtual format could benefit the medical system by limiting the number of missed work days (including on-call responsibilities), reducing strain on the trainee's institution. ${ }^{21}$

Despite these strengths, the biggest perceived weakness among our respondents was limited exposure to the culture/environment of the program. This is of particular relevance to ophthalmology fellowship programs given that the small fellowship class sizes underscore the perceived importance of cultural and program fit which may be limited with a virtual interview process. Although impossible to fully replicate in-person exposure to a program and its city, one study mentioned that it is possible to partially mitigate this by providing interactive virtual tours and designing comprehensive video presentations and online materials. ${ }^{22,23}$

\section{Limitations}

This study has several limitations. Nonresponse bias may be present, as the overall response rate was 34\%. Furthermore, there is also the potential for selection bias. Respondents who had a positive experience at their interview day may have been more likely to respond to our survey. In addition, only applicants applying to Wills Eye Hospital fellowship programs were invited to participate in this survey, and applicants to these fellowship programs may be different than those choosing to apply to other programs. Furthermore, the survey administered was nonvalidated which may limit the reliability of the findings. Lastly, this study evaluated only the preferences of applicants, so the preferences regarding the virtual fellowship interview format by PDs, faculty interviewers, and current residents/fellows were not assessed for comparison.

\section{Conclusion}

In conclusion, this study evaluates perceptions and experiences of ophthalmology fellowship applicants undergoing virtual fellowship interviews in the 2020 to 2021 interview cycle. Presenting these preferences and perceptions may inform how future ophthalmology fellowship interviews occur, regardless of an intervening pandemic. Further study to refine and improve the remote interview format may be of continued interest to medical education training programs.

\section{Meeting Presentation}

None.

Financial Support

None.

\section{Relevant Disclosures}

No conflicting relationship exists for any author. A.J.K. is a consultant for Alimera Sciences, Allergan, and Bausch Health. M.A.K. is a consultant for Genentech and Allergen and a lecturer for Genentech and Regeneron. M.N.C. is a consultant for Allergan.

\section{Conflict of Interest}

None declared

\section{References}

1 Keesara S, Jonas A, Schulman K. Covid-19 and health care's digital revolution. N Engl J Med 2020;382(23):e82

2 Wijesooriya NR, Mishra V, Brand PLP, Rubin BK. COVID-19 and telehealth, education, and research adaptations. Paediatr Respir Rev 2020;35:38-42

3 Johnson J, Chung MT, Stathakios J, Gonik N, Siegel B. The impact of the COVID-19 pandemic on fellowship training: a national survey of pediatric otolaryngology fellowship directors. Int J Pediatr Otorhinolaryngol 2020;136:110217

4 Tseng J. How has covid-19 affected the costs of the surgical fellowship interview process? J Surg Educ 2020;77(05):999-1004

5 Robinson KA, Shin B, Gangadharan SP. A comparison between inperson and virtual fellowship interviews during the covid-19 pandemic. J Surg Educ 2020;78(04):1175-1181

6 Nguyen JK, Shah N, Heitkamp DE, Gupta Y. Covid-19 and the radiology match: A residency program's survival guide to the virtual interview season. Acad Radiol 2020;27(09):1294-1297

7 Nesemeier BR, Lebo NL, Schmalbach CE, et al. Impact of the covid-19 global pandemic on the otolaryngology fellowship application process. Otolaryngol Head Neck Surg 2020;163(04): 712-713

8 Menhaji K, Gaigbe-Togbe BH, Hardart A, et al. Virtual interviews during covid-19: Perspectives of female pelvic medicine and reconstructive surgery program directors. Female Pelvic Med Reconstr Surg 2020

9 Ehrlich H, Boneva D, Mckenney M, Elkbuli A. Virtual interviews for the 2020-2021 national residency matching program during the covid19 pandemic: A curse or blessing? Am Surg 2020;85(04):700-701

10 Hill MV, Ross EA, Crawford D, et al. Program and candidate experience with virtual interviews for the 2020 Complex General Surgical Oncology interview season during the COVID pandemic. Am J Surg 2020:S0002-9610(20)30730-3

11 Susarla SM, Swanson EW, Slezak S, Lifchez SD, Redett RJ. The perception and costs of the interview process for plastic surgery 
residency programs: Can the process be streamlined? Plast Reconstr Surg 2017;139(01):302e-309e

12 Bird SB, Hern HG, Blomkalns A, et al. Innovation in residency selection: The AAMC standardized video interview. Acad Med 2019;94(10):1489-1497

13 Quillen DA, Siatkowski RM, Feldon Sof the Association of University Professors of Ophthalmology. Covid-19 and the ophthalmology match. Ophthalmology 2021;128(02):181-184

14 Christiansen SM, Osher JM, Riemann CD. Association of mentorto-program contact and applicant rank disclosure with vitreoretinal fellowship applicant's final match outcome in 2016 and 2017. JAMA Ophthalmol 2018;136(06):642-647

15 Daram SR, Wu R, Tang SJ. Interview from anywhere: feasibility and utility of web-based videoconference interviews in the gastroenterology fellowship selection process. Am J Gastroenterol 2014;109(02):155-159

16 Edje L, Miller C, Kiefer J, Oram D. Using skype as an alternative for residency selection interviews. J Grad Med Educ 2013;5(03): 503-505
17 Miotto GC. Tele-interview in the aesthetic fellowship selection process. Aesthet Surg J 2018;38(11):NP175-NP177

18 Venincasa MJ, Cai LZ, Gedde SJ, Uhler T, Sridhar J. Current applicant perceptions of the ophthalmology residency match. JAMA Ophthalmol 2020;138(05):460-466

19 Hariton E, Bortoletto P, Ayogu N. Using video-conference interviews in the residency application process. Acad Med 2017;92 (06):728-729

20 Zafar S, Bressler NM, Golnik KC, et al. Fellowship match outcomes in the U.S. from 2010 to 2017: analysis of San Francisco Match. Am J Ophthalmol 2020;218:261-267

21 Pourmand A, Lee H, Fair M, Maloney K, Caggiula A. Feasibility and usability of tele-interview for medical residency interview. West J Emerg Med 2018;19(01):80-86

22 Williams K, Kling JM, Labonte HR, Blair JE. Videoconference interviewing: Tips for success. J Grad Med Educ 2015;7(03):331-333

23 Healy WL, Bedair H. Videoconference interviews for an adult reconstruction fellowship: lessons learned. J Bone Joint Surg Am 2017;99(21):e114 